\section{Angiogenesehemmer neoadjuvant und adjuvant beim operablen Mammakarzinom}

\author{
In der B-40-Studie des National Surgical Adjuvant Breast and Bowel Project \\ (NSABP) wurde der Effekt von Bevacizumab adjuvant und neoadjuvant als \\ Erweiterung chemotherapeutischer Regime bei Patientinnen mit einem \\ operablen, HER2-negativen Mammakarzinom untersucht.
}

\footnotetext{
$\mathrm{n}$ der randomisierten, kontrollierten Phase-III-Studie wurden zwischen 2007 und 20101.206 Brustkrebspatientinnen behandelt. Sie erhielten nach Randomisierung neoadjuvant jeweils eines von drei Docetaxel-basierten Regimen, an die sich jeweils 4 Zyklen AC (Doxorubicin $60 \mathrm{mg} / \mathrm{m}^{2}$ i.v. plus $600 \mathrm{mg} / \mathrm{m}^{2}$ Cyclophosphamid i.v. an Tag $1, \mathrm{q} 3 \mathrm{w})$ anschlossen:

- T $\rightarrow$ AC: Docetaxel $\left(100 \mathrm{mg} / \mathrm{m}^{2}\right.$ i.v. an Tag 1, q3w)

_TX $\rightarrow$ AC: Docetaxel $\left(75 \mathrm{mg} / \mathrm{m}^{2}\right.$ i.v. an

Tag 1, q3w) plus Capecitabin ( $825 \mathrm{mg} /$ $\mathrm{m}^{2}$ p.o. bid, an Tag 1-14) oder

- TG $\rightarrow$ AC: Docetaxel $\left(75 \mathrm{mg} / \mathrm{m}^{2}\right.$ i.v. an

Tag 1, q3w) plus Gemcitabin (1.000

$\mathrm{mg} / \mathrm{m}^{2}$ i.v. an den Tagen $\left.1+8\right)$.
}

Per Studienprotokoll wurden die Patientinnen darüber hinaus für die zusätzliche neoadjuvante und adjuvante Behandlung mit Bevacizumab oder keine zusätzliche antiangiogene Therapie randomisiert. Die Patientinnen mit Bevacizumab-Addition erhielten mit dem ersten neoadjuvanten Chemotherapiezyklus $15 \mathrm{mg} / \mathrm{kg}$ Bevacizumab i.v. (q3w) über 6 Zyklen plus 10 weitere Dosen postoperativ, also adjuvant.

Den zwischen 2007 und 2014 erhobenen Follow-up-Daten zufolge verbesserte weder die Zugabe von Capecitabin noch die von Gemcitabin das krankheitsfreie (DFS) und das Gesamtüberleben (OS). Jedoch verbesserte die Zugabe von Bevacizumab - wie schon in einer früheren Auswertung berichtet - die pathologische Komplettremissionsrate von Frauen mit einem operablen, invasiven, nicht metastasierten HER2-negativen Mammakarzinom. Bevacizumab neoadjuvant und adjuvant zusätzlich zum Chemotherapieregime erhöht auch signifikant den sekundären Endpunkt Gesamtüberlebensrate, nicht jedoch das krankheitsfreie Überleben.

Fazit: Frauen mit einem operablen, HER2-negativen Mammakarzinom erfahren keinen Nutzen von der Erweiterung einer neoadjuvanten Chemotherapie auf Basis von Docetaxel/Doxorubicin/Cyclophosphamid um Gemcitabin oder Capecitabin. Der in dieser Studie gezeigte Überlebensvorteil durch Bevacizumab in der neoadjuvanten und adjuvanten Therapie verdient es nach Ansicht der Forscher weiter untersucht zu werden.

Wolfgang Zimmermann

Bear HD et al. Neoadjuvant plus adjuvant bevacizumab in early breast cancer (NSABP B-40 [NRG Oncology]): secondary outcomes of a phase 3, randomised controlled trial. Lancet Oncol. 2015;16(9):1037-48.

\title{
Letrozol wirkt anders als Tamoxifen - besonders bei lobulärem Brustkrebs
}

\section{Anhand der Daten der BIG 1-98- Studie wurde retrospektiv die Effek- tivität von Letrozol und Tamoxifen bei Patientinnen mit invasiven duktalen oder lobulären Mamma- karzinomen verglichen.}

nsgesamt 2.923 Frauen mit frühem invasivem duktalem (IDC; $\mathrm{n}=2.599$ ) oder klassischem invasivem lobulärem Karzinom (ILC; $\mathrm{n}=324$ ) hatten randomisiert entweder Letrozol oder Tamoxifen adjuvant erhalten. Per Ki-67-Status wurden bei IDC und ILC zusätzlich Tumoren mit hoher (Luminal B) oder niedriger (Luminal A) Proliferationsaktivität unterschieden.

Nach median 8,1 Jahren zeigte sich, dass der Nutzen der Therapie signifikant von der Histologie des Tumors (duktal/ lobulär) abhing. Das krankheitsfreie 8-Jahres-Überleben (DFS) war unter Letrozol höher, sowohl bei Frauen mit ILC
(82 vs. $66 \%$; Hazard Ratio [HR] 0,48), als auch bei solchen mit IDC ( 82 vs. $75 \%$; HR 0,80). Auch das 8-Jahres-Gesamtüberleben (OS) war unter Letrozol in der ILC-Gruppe (89 vs. $74 \%$; HR 0,40) und in der IDC-Gruppe (88 vs. $84 \%$; HR $0,73)$ besser. Darüber hinaus spielte die Proliferationsaktivität (Ki-67), enthalten in der Klassifikation Luminal A oder Luminal B, eine Rolle.

Diese Ergebnisse bestätigten sich in der multivariaten Analyse. Hier zeigten sich für das DFS signifikante Interaktionen zwischen dem Nutzen der Therapie mit Letrozol im Vergleich zu Tamoxifen und der Histologie (ILC oder IDC; $\mathrm{p}=0,006)$.

Auch die Klassifikation als Luminal A oder Luminal B hatte einen signifikanten Einfluss auf die Wirksamkeit der Letrozol-Gabe im Vergleich zu Tamoxifen $(p=0,01)$. Bei den Frauen mit lobulären Karzinomen der Untergruppe Luminal
B (ILC/LB) zeigte sich unter Letrozol eine im Vergleich zu Tamoxifen um $66 \%$ reduzierte Wahrscheinlichkeit eines DFS-Ereignisses (HR 0,34), für die Gruppe ILC/LA eine 50\%ige Reduktion (HR 0,50). Bei den invasiven duktalen Karzinomen mit LB-Subtyp (IDC/LB) ergab sich eine um $35 \%$ reduzierte Wahrscheinlichkeit eines DFS-Ereignisses (HR 0,65). Nur beim invasiven duktalen Karzinom vom Luminal-A-Subtyp (IDC/LA) war kein signifikanter Nutzen von Letrozol versus Tamoxifen hinsichtlich des DFS festzustellen.

Fazit: Der Nutzen von Letrozol im Vergleich zu Tamoxifen ist bei lobulären Mammakarzinomen größer als bei invasiven duktalen.

Brigitte Schalhorn

Metzger Filho $\mathrm{O}$ et al. Relative effectiveness of letrozole compared with tamoxifen for patients with lobular carcinoma in the BIG 1-98 trial. J Clin Oncol. 2015;33(25):2772-9. 